\title{
Label Free Biochemical Determinations Based on the Contrast Monitoring of Periodic Patterns
}

\author{
Ioannis Archontas, Alexandros Salapatas, Konstantinos Misiakos \\ Microelectronics Institute, NCSRD Demokritos, 15310, Athens, Greece \\ misiakos@imel.demokritos.gr
}

\begin{abstract}
:
A method is presented for label-free detection of protein-protein binding in real time. It is based on the protein induced contrast modulation of the microscope image of an oxidized and patterned silicon substrate. Interferometric effects make the reflection coefficient of the solution-protein-oxide-silicon stack a function of the oxide-protein combined thickness. As a consequence, the contrast of the pattern changes during the experiment and the magnitude of the change is a measure of the progress of the biomolecular reaction. A model bioanalytical assay demonstrates the potential of the method.
\end{abstract}

Key words: optical, biosensors, Fourier transform, signal processing

\section{Direct biochemical sensing through signal processing of digital microscope pictures}

Optical detection in bioanalytical systems is superior to other types of sensing owing to the galvanic isolation of the transducer from the excitation and detection electronics [1]. Two factors play critical role in expanding the use of label free and real time assays through optical systems: The use of existing equipment with minimal modifications or additions, as well as the capability for multianalyte determinations. Here, we demonstrate a method for protein binding monitoring based on the protein induced contrast modulation of the digital microscope image of a patterned substrate. The method is label free, has multianalyte potential and requires only a digital microscope, as far as optics is concerned. A silicon wafer with a grown 100nm silicon dioxide was employed as the solid support for the biomolecules. The wafer was patterned so that a chessboard geometry was created with square oxide islands surrounded by bare silicon. After coating with biotinylated BSA, it was inserted in a fluidic structure and placed under the microscope while a micropump was supplying streptavidin solutions. The microscope light was filtered so that blue light $(425-475 \mathrm{~nm})$ was impinging and reflected on the wafer. The reflection coefficient of the solution-proteinoxide-silicon stack is a function of the oxideprotein combined thickness for a specific spectral range. For the parameters chosen, the reflectivity shows maximum sensitivity on the protein adlayer, as shown in Fig.1. On the other hand, on bare silicon where the oxide was removed the reflection coefficient is at its maximum value and barely changes upon binding. As a consequence, the contrast of the chessboard pattern changes during the experiment and the quantified change monitors the progress of the biomolecular reaction.

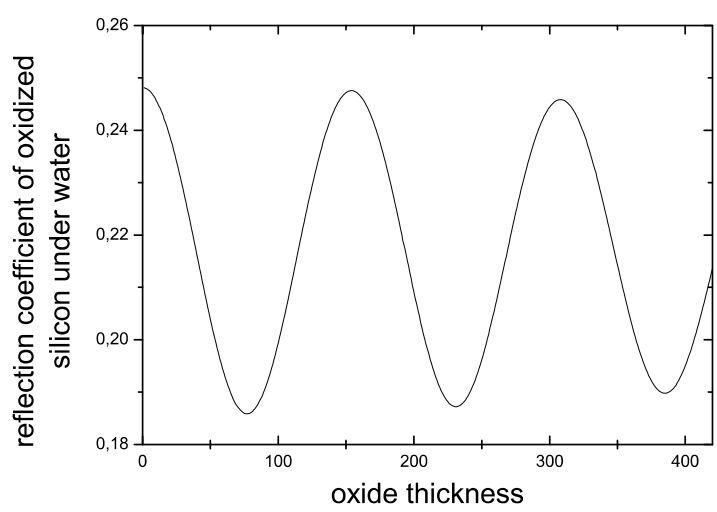

Fig. 1. Average reflection coefficient in the 425-475 $\mathrm{nm}$ range as a function of the $\mathrm{SiO}_{2}$ thickness. The substrate is silicon and the superstrate is water.

\section{Protein assay results}

The wafer was placed in a fluidic structure consisting of two Al plates, a quartz plate, a PDMS gasket, and plastic tubing (Fig. 2). The top Al plate had a quartz window so that the silicon oxide pattern could be monitored on real time while biomolecular solutions were pumped through the fluidic channels. Four screws were holding the two Al plates in place and provided 
the required pressure so that the PDMS gasket would seal the fluidic channel. The monitoring was performed by placing the structure on the microscope chuck, focusing through the quartz window on the patterned silicon surface and by filtering the microscope light in the blue (426475nm, Fig. 2a).

Digital signal processing through 2-D Fourier transform is employed to isolate the contrast factor at a good enough resolution. Such a result is shown in Fig. 3 . In the first 100 picture shots, where only the buffer solution flows in, the signal is stable. The magnitude of the Fourier transform sharply declines for the next 100 shots and the starts leveling off after 200 shots (800 seconds). This is due to the saturation of the binding sites on the silicon wafer. In any case a contrast drop of about $30 \%$ is obtained. The signal to noise ratio is high and problems only arise when external factors (for instance a bubble as in shot number 330) interfere.

The two dimensional images shown in Fig. 4 illustrate the reflectance of the chessboard pattern at the various points before and after the reaction. The blue squares are the silicon oxide square areas where the reflectance is lower compared to the reflectance of the surrounding bare silicon. The contrast shown is larger before the reaction because of the lower reflectance oxide regions (deep blue areas, Fig. 4a) compared to the same after the reaction (blue areas, Fig. 4b).

The above described method for the monitoring of biomolecular binding can be extended to cover cases where a number of different probes have been immobilized on silicon leading to an equal number of different reactions taking place simultaneously. In this case the field to be illuminated and continuously monitored is segmented into the corresponding spotting areas, while the algorithm is modified so that each area is independently processed through Fourier transform to isolate the contrast factor for each different spot. In such a case of course, the space bit count per area will be reduced resulting in higher noise levels.

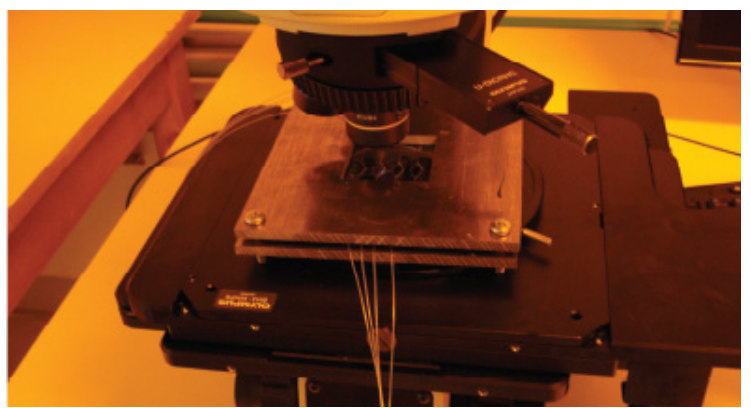

(a)

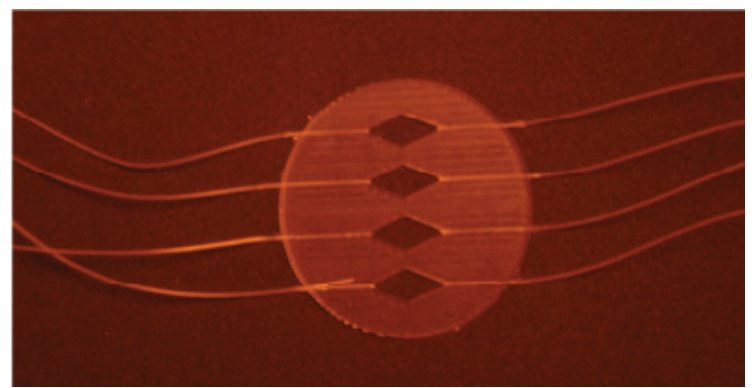

(b)

Fig. 2. Fluidic structure under the microscope and PDMS gasket. Two aluminum plates hold together a quartz plate, a PDMS gasket and the patterned silicon wafer (a). The PDMS fluidic gasket made by mold casting. The polymer fluidic tubings are clearly seen. Also seen are the diamond shaped openings that form the reaction chambers after being sandwiched between the quartz plate and the silicon wafer (b).

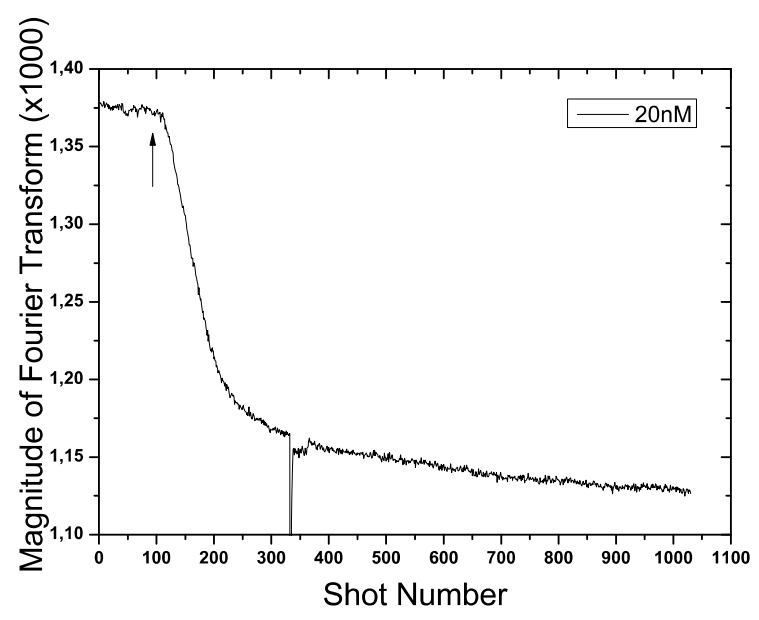

Fig. 3. Magnitude of the Fourier transform as a function of shot number (picture number) in the case of $20 \mathrm{nM}$ streptavidin reaction with the immobilized biotinylated BSA. Every shot is taken every 4 seconds. From 0 to 100 the buffer solution flows in and then (arrow) a $20 \mathrm{nM}$ streptavidin solution in the same buffer is introduced. 


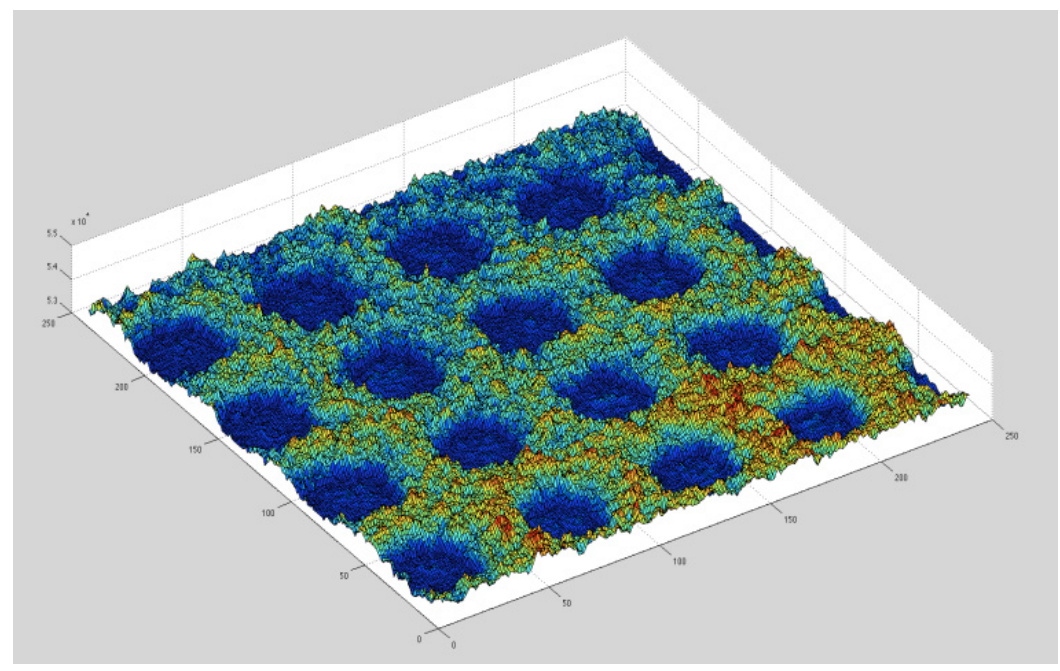

(a)

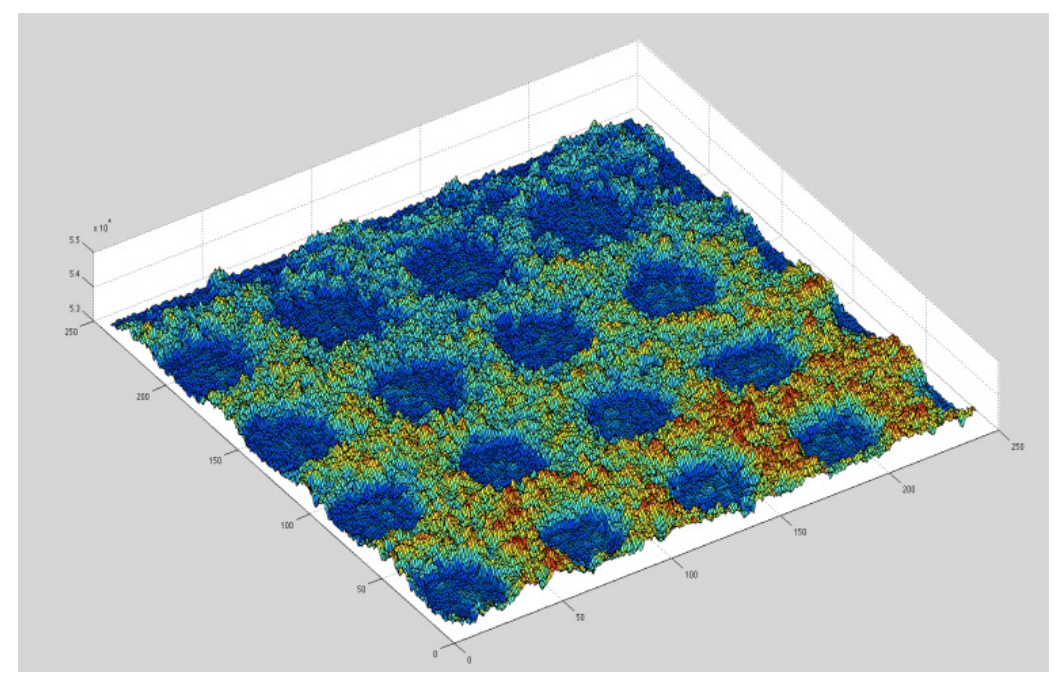

(b)

Fig. 4. Two dimensional plots of the reflectance spatial distribution before (a) and after (b) the reaction with streptavidin (20 nM streptavidin solution, reaction took 4000 seconds)

\section{Conclusions}

Label free protein-protein binding is monitored in real time by taking advantage of the protein induced contrast modulation in the microscope image of an oxidized and patterned silicon substrate. The pattern consists of silicon oxide squares surrounded by bare silicon areas. The reflection coefficient of the solution-proteinoxide-silicon stack is a function of the oxideprotein combined thickness. The oxide thickness is selected, in conjunction with the spectral range employed, so that it presents maximum sensitivity with regard to reflection coefficient dependence on the added protein layer. The bare silicon surface, on the other hand, shows close to zero sensitivity as far as its reflectivity is concerned. As a consequence, the contrast of the bio- functionalized chessboard pattern changes during binding and the change is used as a measure of the progress of the biomolecular reaction. To isolate the contrast of the pattern, the digital files were processed through two dimensional fast Fourier transform to calculate the magnitude of the Fourier signal at the point of maximum in the transform domain. This particular digital signal processing technique is immune to random fluctuations of the signal and offset levels and depends primarily in the contrast of the periodic pattern. Multianalyte functionality is possible if the illuminated field is segmented into areas where different biomolecular probes have been immobilized.

\section{References}

[1] Turner, A. P. F. "Biosensors-sense and sensitivity”, Science 290, 1315-1317, 2000. 